\section{Loss-of-function mutations in FGFR1 cause autosomal dominant Kallmann syndrome}

\author{
Published online 10 March 2003; doi:10.1038/ng1122
}

\begin{abstract}
We took advantage of overlapping interstitial deletions at chromosome 8p11-p12 in two individuals with contiguous gene syndromes and defined an interval of roughly $540 \mathbf{~ k b}$ associated with a dominant form of Kallmann syndrome, KAL2. We establish here that loss-of-function mutations in FGFR1 underlie KAL2 whereas a gain-of-function mutation in FGFR1 has been shown to cause a form of craniosynostosis. Moreover, we suggest that the KAL1 gene product, the extracellular matrix protein anosmin-1, is involved in FGF signaling and propose that the gender difference in anosmin-1 dosage (because KAL1 partially escapes $\mathrm{X}$ inactivation) explains the higher prevalence of the disease in males.
\end{abstract}

Kallmann syndrome involves hypogonadotropic hypogonadism and anosmia, a deficiency of the sense of smell. Anosmia is related to the absence or hypoplasia of the olfactory bulbs and tracts. Hypogonadism is due to deficiency in gonadotropinreleasing hormone $(\mathrm{GnRH})$ and probably results from a failure of embryonic migration of neurons that synthesize GnRH. These cells normally migrate from the olfactory epithelium to the forebrain along the olfactory nerve pathway ${ }^{1}$. Familial cases of Kallmann syndrome have been reported $^{2-4}$, which suggested X-chromosome linked, autosomal dominant, or, less often, recessive modes of inheritance of the disease. Two intriguing points have been noted: sporadic cases are much more numerous than familial cases, and the disease has a much higher prevalence in males $(1: 10,000)$ than in females (the sex ratio has been estimated at 5:1). The gene underlying the $\mathrm{X}$-linked form of the disease (KAL1) has been identified ${ }^{1}$. But in many families with only males affected and presumably maternal transmission of the disease, no mutation has been found in the KAL1 coding region. Moreover, a molecular analysis of the gene in sporadic male cases rarely identified a mutation (ref. 4 and C.D., unpublished results). This suggests that mutations in the $\mathrm{X}$-linked gene KAL1 do not account for the higher prevalence of the disease in males. Because the incomplete penetrance of the disease $\mathrm{e}^{2,3}$ and the infertility in affected individuals impede linkage analysis, the genes underlying the autosomal forms of Kallmann syndrome have so far escaped identification.

By segregation analysis of polymorphic markers combined with fluorescence in situ hybridization (FISH) analysis, we determined that two de novo deletions of roughly $10.7 \mathrm{Mb}$ and $11.6 \mathrm{Mb}$ at chromosome 8 p11.2-p12 in two individuals ${ }^{5}$ who are affected by different contiguous gene syndromes that both include Kallmann syndrome overlap by approximately $540 \mathrm{~kb}$ (see Supplementary Notes 1 and 2 and Supplementary Fig. 1 online). Three genes have been reported in this interval; from telomere to centromere, they are WHSC1L1 (OMIM 607083), FGFR1 (OMIM 136350) and the first two exons of TACC1 (OMIM 605301). FGFR1 encodes the fibroblast growth factor receptor 1 and was considered the best candidate to underlie Kallmann syndrome because of a possible functional interaction with the KAL1 gene product.

Southern-blot analysis of 43 individuals with familial or sporadic Kallmann syndrome using two genomic probes encompassing exons 4-5 and 16-18 of FGFR1 did not show a deletion of the gene in any of them (data not shown). We then determined the nucleotide sequence of the 18 coding exons and splice sites of FGFR1 in 129 unrelated individuals with Kallmann syndrome (91 males and 38

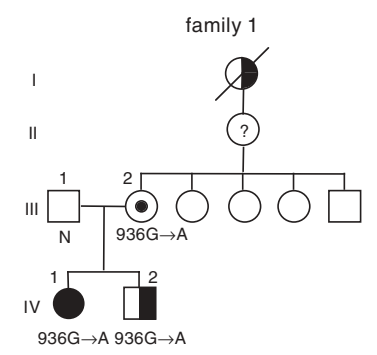
unknown clinical status. females). In four familial cases (Fig. 1) and eight sporadic cases, we detected heterozygous mutations of FGFR1: one nonsense mutation, two frameshift mutations, two donor splice-site mutations and seven missense mutations. In addition, we found that one individual, who was born to consanguineous parents and is affected by Kallmann syndrome with cleft palate, agenesis of the corpus callosum, unilateral hearing loss and fusion of the fourth and fifth metacarpal bones, was homozygous with respect to a deleterious missense mutation (Table 1 and Supplementary Fig. 2 online). From these results, we conclude that FGFR1 underlies one autosomal dominant form of Kallmann syndrome (KAL2). Moreover, cleft palate or lip and dental agenesis, two anomalies that are occasionally associated with Kallmann syndrome $\mathrm{e}^{1-3}$ and were present in five individuals with mutations in FGFR1 (Table 1), can now be ascribed to this genetic form of the disease. We also found that bimanual synkinesia, identified in one family with KAL2 (Table 1), can be observed in autosomal Kallmann syndrome, whereas it had thus far been considered to be specific to the X-linked form of the disease.

FGF signaling is involved in a variety of developmental processes including the formation, growth and shaping of tissues and organs. Extracellular interaction between FGF, the FGF receptor and heparan sulfate proteoglycans (HSPGs) is required for receptor dimerization and resulting autophosphorylation of several tyrosine residues in the intracellular domain. The phosphotyrosines either stimulate protein tyrosine kinase activity of the receptor or serve as docking sites for downstream signaling molecules. A
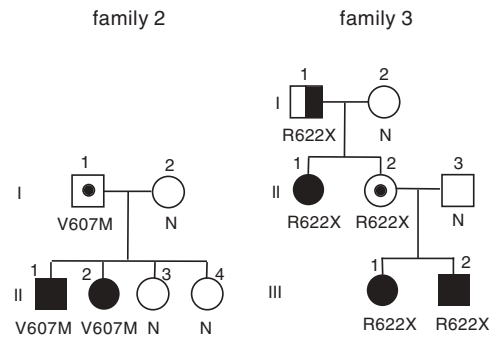

family 4

Fig. 1 Segregation of the mutations in FGFR1 in four families affected with Kallmann syndrome. Filled symbols indicate clinically affected individuals with both hypogonadism and anosmia. Half-filled symbols indicate individuals with anosmia only. Open symbols containing a black dot indicate unaffected carriers of the mutation. $\mathrm{N}$ indicates absence of the mutation. In family 1, individual IV2 (who was 16 years old) underwent spontaneous puberty but suffers from anosmia; individuals III2, IV1 and IV2 have multiple dental agenesis. In family 2, individuals II1 and II2 both have bimanual synkinesia. In family 3, individual III1 has a cleft lip and individual III 2 has both a cleft lip and a cleft palate. Notably, non-penetrance of the disease in some mutation carriers can simulate recessive transmission of Kallmann syndrome in certain families, for example, families 1, 2 and 4. Diagonal line indicates deceased individual; question mark indicates 
Table 1 - Mutations in FGFR1 associated with Kallmann syndrome

\begin{tabular}{|c|c|c|}
\hline Mutation & Exon/Intron & Kallmann syndrome associated symptoms \\
\hline G97D & exon 3 & \\
\hline Y99C & exon 3 & \\
\hline 303-304insCC & exon 3 & \\
\hline A167S & exon 5 & $\begin{array}{l}\text { cleft palate, corpus callosum agenesis, unilateral hearing loss, fusion of } \\
\text { the fourth and fifth metacarpal bones }\end{array}$ \\
\hline C277Y & exon 7 & \\
\hline $936 \mathrm{G} \rightarrow \mathrm{A}$ & exon 7 (donor splice site) & multiple dental agenesis \\
\hline V607M & exon 13 & bimanual synkinesis \\
\hline $\mathrm{R} 622 \mathrm{X}$ & exon 14 & cleft lip or palate \\
\hline 1970-1971delCA & exon 14 & \\
\hline W666R & exon 15 & cleft palate \\
\hline IVS15+1G $\rightarrow$ A & intron 15 (donor splice site) & \\
\hline M719R & exon 16 & \\
\hline P772S & exon 18 & cleft palate, unilateral absence of nasal cartilage, iris coloboma \\
\hline \multicolumn{3}{|c|}{ 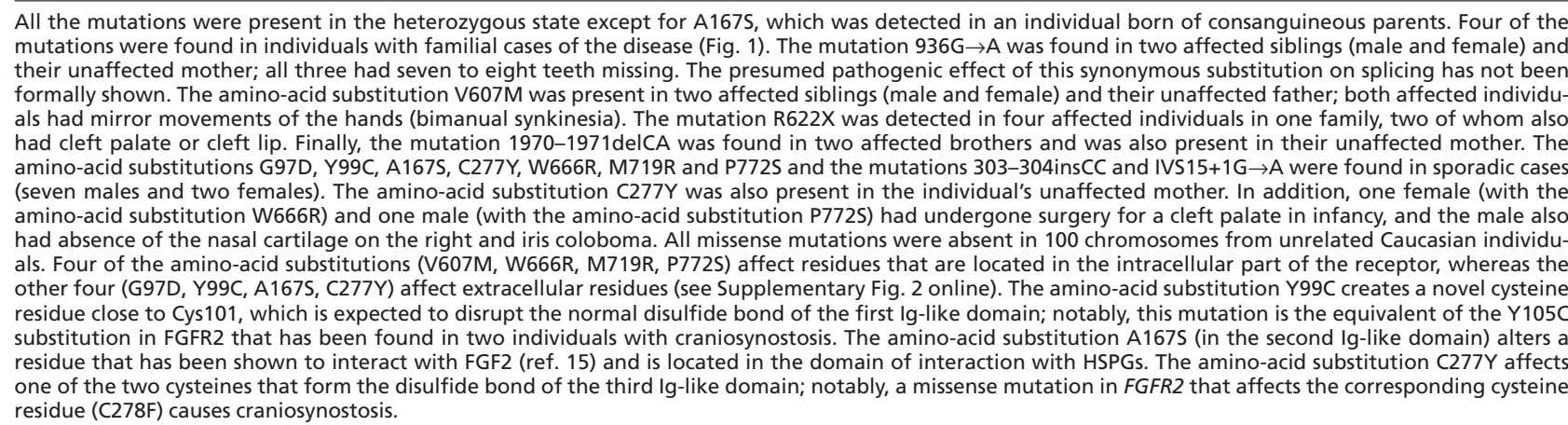 } \\
\hline
\end{tabular}

dominant gain-of-function mutation of FGFR1 underlies a form of craniosynostosis $^{6}$. This mutation is believed to result in enhanced affinity for certain FGF ligands ${ }^{7}$. In contrast, KAL2 results from loss-of-function mutations in FGFR1, as shown by the presence of a single allele in the individuals carrying a chromosomal deletion at 8 p11.2. Moreover, some of the mutations associated with KAL2 could hinder the formation of functional receptor dimers by a dominant negative effect. Whatever the molecular mechanism may be, our results indicate that olfactory bulb development in humans is crucially sensitive to reduced dosage of FGFR1. Dominant gain-of-function and loss-of-function mutations in FGFR1 cause different developmental disorders: premature fusion of skull bone sutures (craniosynostosis) and failed morphogenesis of the olfactory bulbs (Kallmann syndrome), respectively. Given the phenotype associated with gain-of-function mutation of FGFR1, it is noteworthy that delayed closure of calvarial sutures has not been reported in Kallmann syndrome.

The implication of FGFR1 and KAL1 in the same developmental disease raises the possibility that the gene products functionally interact. KAL1 encodes anosmin-1, a locally restricted protein of roughly $100 \mathrm{kDa}$ in embryonic extracel- lular matrices ${ }^{8}$. Several arguments suggest that anosmin-1 is involved in FGF signaling through FGFR1. First, anosmin-1 binds to HSPGs ${ }^{9}$, and HSPGs are important in the dimerization of the binary FGF-FGFR complex ${ }^{10}$. Also, the axon-branching phenotype in some neurons in Caenorhabditis elegans that misor overexpress the nematode gene kall was no longer present in mutant worms lacking heparan 6O-sulfotransferase, an enzyme involved in HSPG biosynthesis $^{11,12}$. Second, KAL1 and FGFR1 are coexpressed at different sites during embryonic development. KAL1 is expressed in the presumptive olfactory bulbs $^{8}$, and $F g f r 1$ expression in the rostral forebrain is required for initial olfactory bulb evagination in the mouse ${ }^{13}$. In addition, cleft palate or cleft lip was present in several individuals with KAL2 (Table 1), and a high arched palate, which can be regarded as a mild anomaly of palatal fusion, is a common feature of KAL1. Finally, the bimanual synkinesia that we report in one family with KAL2 has been observed in $75 \%$ of KAL1 cases.

Regarding the low prevalence of the Xlinked form of the disease ${ }^{4}$, the proposal that KAL1 and FGFR1 functionally interact provides a possible explanation for the higher prevalence of Kallmann syndrome in males. The explanation is based on the assumption that the local concentration of anosmin-1 is important in FGF signaling and the fact that KAL1 partially escapes $\mathrm{X}$ inactivation in females ${ }^{14}$. Accordingly, females are expected to synthesize a higher amount of anosmin-1 than do males; in some cases, this could be enough to maintain FGF signaling above the critical threshold in the context of FGFR1 haploinsufficiency. Indeed, in four of five families in whom we were able to follow the transmission of the FGFR1 mutation, the mutation was transmitted by the unaffected mother (Table 1). Moreover, the probable pseudoautosomal location of Kal1 in rodents can account for the observation that male mice heterozygous with respect to a null allele at the Fgfr 1 locus are viable and fertile ${ }^{13}$.

Note: Supplementary information is available on the Nature Genetics website.

\section{Acknowledgments}

We thank the individuals with Kallmann syndrome and their families for their participation in this study, the Service de séquençage de l'Institut Cochin and J. Weissenbach at the Centre National de Séquençage for sequencing facilities, $B$. Menten and $E$. Hermanci for expert technical assistance, J. Hébert and S. McConnell for sharing results before publication and S. Cure for critical reading 
of the manuscript. This study was supported by European Community, Human Frontiers Science Program (Génétique des Déficits Sensoriels, Paris, France) and Fund for Scientific ResearchFlanders (Belgium).

\section{Competing interests statement}

The authors declare that they have no competing financial interests.

Catherine Dodé ${ }^{1}$, Jacqueline Levilliers ${ }^{2}$, Jean-Michel Dupont ${ }^{3}$, Anne De Paepe ${ }^{4}$, Nathalie Le Dû ${ }^{3}$, Nadia Soussi-

Yanicostas $^{2}$, Roney S. Coimbra ${ }^{2}$, Sedigheh Delmaghani², Sylvie CompainNouaille $^{2}$, Françoise Baverel ${ }^{3}$, Christophe Pêcheux ${ }^{1}$, Dominique Le Tessier ${ }^{3}$, Corinne Cruaud ${ }^{5}$, Marc Delpech $^{1}$, Frank Speleman ${ }^{4}$, Stefan Vermeulen ${ }^{4}$, Andrea Amalfitano ${ }^{6}$, Yvan Bachelot ${ }^{7}$, Philippe Bouchard ${ }^{8}$, Sylvie Cabrol ${ }^{9}$, Jean-Claude Carel ${ }^{10}$, Henriette Delemarre-van de Waal ${ }^{11}$, Barbara Goulet-Salmon ${ }^{12}$, Marie-Laure Kottler ${ }^{13}$, Odile Richard ${ }^{14}$,
Franco Sanchez-Franco ${ }^{15}$, Robert Saura ${ }^{16}$, Jacques Young ${ }^{17}$, Christine Petit ${ }^{2}$ \& Jean-Pierre Hardelin ${ }^{2}$

${ }^{1}$ Institut Cochin et Laboratoire de Biochimie et Génétique Moléculaire, Hôpital Cochin, 75014 Paris, France. ${ }^{2}$ Unité de Génétique des Déficits Sensoriels, Institut Pasteur, 75724 Paris cedex 15, France. ${ }^{3}$ Institut Cochin et Laboratoire de Cytogénétique, Hôpital Cochin, Paris, France. ${ }^{4}$ Center for Medical Genetics, Ghent University Hospital, Ghent, Belgium. ${ }^{5}$ Génoscope, Évry, France. ${ }^{6}$ Duke University Medical Center, Durham, North Carolina, USA. ${ }^{7}$ Hôpital A. Michallon, Grenoble, France. ${ }^{8}$ Service d'Endocrinologie, Hôpital Saint-Antoine, Paris, France. ${ }^{9}$ Service de Physiologie, Hôpital Armand Trousseau, Paris, France. ${ }^{10}$ Service d'Endocrinologie Pédiatrique, Hôpital SaintVincent de Paul, Paris, France. ${ }^{11}$ Department of Pediatrics, VU University Medical Center, Amsterdam, the Netherlands. ${ }^{12}$ Centre hospitalier d'Alençon, Alençon, France. ${ }^{13}$ Département Génétique et Reproduction, Hôpital Clemenceau, Caen, France. ${ }^{14}$ Service de Pédiatrie et Génétique, Hôpital Nord, SaintÉtienne, France. ${ }^{15}$ Instituto de Salud Carlos III, Madrid, Spain. ${ }^{16}$ Service de Génétique
Médicale, Hôpital Pellegrin, Bordeaux, France. ${ }^{17}$ Service d'Endocrinologie, Hôpital de Bicêtre, Le Kremlin-Bicêtre, France. Correspondence should be addressed to J.-P.H (e-mail: hardelin@pasteur.fr).

Received 22 November 2002; accepted 6 February 2003.

1. Hardelin, J.-P. Mol. Cell. Endocrinol. 179, 75-81 (2001).

2. Santen, R.J. \& Paulsen, C.A. J. Clin. Endocrinol. Metab. 36, 47-54 (1972).

3. White, B.J., Rogol, A.D., Brown, K.S., Lieblich, J.M. \& Rosen, S.W. Am. J. Med. Genet. 15, 417-436 (1983).

4. Oliveira, L.M.B. et al. J. Clin. Endocrinol. Metab. 86, 1532-1538 (2001).

5. Vermeulen, S. et al. Am. J. Med. Genet. 108, 315-318 (2002).

6. Muenke, M. et al. Nat. Genet. 8, 269-274 (1994).

7. Anderson, J., Burns, H., Enriquez-Harris, P., Wilkie, A. \& Heath, J. Hum. Mol. Genet. 7, 1475-1483 (1998).

8. Hardelin, J.-P. et al. Dev. Dyn. 215, $26-44$ (1999).

8. Hardelin, J.-P. et al. Dev. Dyn. 215, 26-44 (1999). (1996).

10. Pellegrini, L. Curr. Opin. Struct. Biol. 11, 629-634 (2001).

11. Bülow, H.E., Berry, K.L., Topper, L.H., Peles, E. \& Hobert, O. Proc. Natl. Acad. Sci. USA 99, 6346-6351 (2002).

12. Soussi-Yanicostas, N. et al. Cell 109, 217-228 (2002)

13. Hébert, J.M., Partanen, J., Rossant, J. \& McConnell, S.K. Development 130, 1101-1111 (2003).

14. Franco, B. et al. Nature 353, 529-536 (1991).

15. Plotnikov, A.N., Schlessinger, J., Hubbard, S.R. \& Mohammadi, M. Cell 98, 641-650 (1999). 